\title{
Fermilab
}

FERMILAB-Conf-01/002-E CDF January 2001

\section{STANDARD MODEL HIGGS COMBINED RESULT FROM CDF}

\author{
JOĀO GUMMARÄES DA COSTA \\ Department of Physics, Harvard University \\ Cambridge, Massachussets 08138, USA \\ (On behalf of the CDF Collaboration)
}

\begin{abstract}
The Tevatron is expected to be most sensitive to the Standard Model Higgs in its associated production with a $\mathrm{W}$ or $\mathrm{Z}$ boson. The Collider Detector at Fermilab (CDF) has performed individual searches for such production in each decay channel of the vector boson, assuming that the Higgs decays to $b \bar{b}$. These searches tse data collected by CDF during the 1992-95 run. The individual results are reviewed, and a combined cross scction limit is presented.
\end{abstract}

The Standard Model (SM) has been remarkably successful in explaining the last decade of precision electroweak measurements, however, the dynamies responsible for the electroweak symmetry breaking remains unknown. The simplest model for this mechanism is spontaneous symmetry breaking achieved through the introduction of a single scalar particle, the Higgs boson. A global fit to the current electroweak data gives a $95 \%$ C.L. upper limit on the Higgs mass of $165 \mathrm{GeV} / \mathrm{c}^{2}$.

The dominant production mechanism of the SM Higgs boson at the Tevatron is $g g \rightarrow H$. This mode has extremely high QCD backgrounds if one searches for the light Higgs main decay mode, $H \rightarrow b \bar{b}$. Thus, the most sensitive production channels at the Tevatron are the ones with an associated vector boson, $q \vec{q}^{-} \rightarrow V^{*} \rightarrow V H$, where the vector boson, either a $W$ or a $Z$, provides a bandle to reduce the QCD background. The CDF collaboration has searched for $V H$ production using the data collected from 1992 through 1995 . Individual searches have been performed in all the vector boson decay channels except those involving taus: $W H \rightarrow l \nu b \bar{b}$, $W H / Z H \rightarrow q g b \bar{b}, Z H \rightarrow \nu \bar{\nu} b \bar{b}$ and $Z H \rightarrow l^{+} l^{-} b \bar{b}$, with $l=e$ or $\mu$. The results are summarized in Table 1.

The search strategy is similar for every decay channel. We start by identifying the presence of the vector boson and require at least two extra jets from the Higgs decay. The ability to identify $b$ jets with a large efficiency and a low mistag rate is vital for searching for the $H^{0} \rightarrow b \bar{b}$ decay. We use three b-tagging algorithms: secondary vertex, soft-lepton and jet probability. ${ }^{2}$ All analysis require at least one jet to be tagged by the secondary vertex algorithm, which has a higher signal-tonoise ratio. The data is separated in independent samples with one or two b-tags and we perform a direet search for a resonant mass peak in the dijet mass spectrum.

In the $W H \rightarrow l \nu b \bar{b}$ channel, a $W$ boson sample is selected by requiring an isolated high- $P_{T}$ electron (muon) with $E_{T}>20 \mathrm{GeV}\left(P_{T}>20 \mathrm{GeV} / c\right)$ and $|\eta|<1.0$ 
Table 1. Acceptance for three Higgs masses; integrated luminosity, expected background and observed events for the different $W$ decay channels.

\begin{tabular}{|c|c|c|c|c|c|c|c|}
\hline \multicolumn{2}{|c|}{$\begin{array}{c}\text { Channel } \\
b \text {-tags }\end{array}$} & \multicolumn{3}{|c|}{$A_{V H}(\%), M_{H}\left(\mathrm{GeV} / \mathrm{c}^{2}\right)$} & $\begin{array}{c}\text { Luminosity } \\
\left(p b^{-1}\right)\end{array}$ & $\begin{array}{l}\text { Background } \\
\text { predicted }\end{array}$ & $\begin{array}{l}\text { Observed } \\
\text { events }\end{array}$ \\
\hline lvbb & 1 & $.55 \pm .14$ & $.74 \pm .18$ & $.89 \pm .22$ & 106 & $30 \pm 5$ & 36 \\
\hline$i v b \bar{b}$ & 2 & $.23 \pm .06$ & $.29 \pm .07$ & $.34 \pm .09$ & 106 & $3.0 \pm .6$ & 6 \\
\hline$q q b \bar{b}$ & $\mathbf{2}$ & $1.3 \pm .4$ & $2.2 \pm .6$ & $3.1 \pm 0.8$ & 91 & $594 \pm 30$ & 589 \\
\hline$\nu \bar{D} b \bar{b}$ & 1 & $.59 \pm .12$ & $.69 \pm .14$ & $.86 \pm .17$ & 88 & $39.2 \pm 4.4$ & 40 \\
\hline$\nu \bar{\nu} b \bar{b}$ & 2 & $.37 \pm .08$ & $.44 \pm .11$ & $.53 \pm .11$ & 88 & $3: 9 \pm .6$ & 4 \\
\hline$u b \bar{b}$ & $\geq 1$ & $.14 \pm .03$ & $.20 \pm .04$ & $.19 \pm .04$ & 106 & $3.2 \pm .7$ & 5 \\
\hline
\end{tabular}

and missing transverse energy $\left(\phi_{T}\right)$ larger than $20 \mathrm{GeV} .^{3}$ Dilepton events consistent with $t \bar{t}$ or $Z^{\circ}$ decay are removed. We select events with two jets with $E_{T}>15 \mathrm{GeV}$ and $|\eta|<2.0$ and require that one or both jets be identified as coming from a $b$ hadron. We require at least one jet to be tagged by the secondary vertex algorithm. In the double-tag analysis, the second jet can also be tagged by the soft lepton $b$-tagging algorithm. The major sources of background are direct production of $W$ bosons in association with heavy flavor $(W \bar{b} \bar{b}, W c \bar{c}, W c)$, mistags due to track mismeasurements and $t \bar{t}$ and single top production.

In the $W H / Z H \rightarrow q q b \bar{b}$ channel, the events are required to have four or more jets with $E_{T}>15 \mathrm{GeV}$ and $|\eta|<2.4$ At least two among the four highest- $E_{T}$ jets must be identified as $b$ quarks by the secondary vertex algorithm. The mass reconstruction is done with the four highest- $E_{T}$ jets, with two highest- $E_{T} b$-tagged jets assigned to the Higgs boson. A cut on $P_{T}(b \bar{b}) \geq 50 \mathrm{GeV} / c$ reduces greatly the QCD background due to direct production and flavor excitation of heavy quarks. Other backgrounds are $t \bar{t}$ production, $Z$ plus heavy flavor with $Z \rightarrow b \bar{b} / c \bar{c}$ and fake double tags.

The $Z H \rightarrow \nu \bar{\nu} b \bar{b}$ is the most sensitive analysis because of the relative lower backgrounds when compared with $Z \rightarrow q \bar{q}$. We select events with a large missing transverse energy, $\mathscr{F}_{T}>40 \mathrm{GeV}$ and two or three jets with $E_{T}>15 \mathrm{GeV}$ and $|\eta|<2$. The QCD background is reduced by removing events whose leading jets are back to back, $\Delta \phi\left(j_{1}, j_{2}\right)<150^{\circ}$, and events with soft jets close in $\phi$ to the $t_{T}$ direction, $\Delta \phi\left(j, H_{T}\right) \geq 60^{\circ}$. We make independent analysis with single and double $b$-tagged samples, using the jet probability algorithm in the double-tagged sample. The dijet invariant mass distributions are shown in Figurc 1. The major remaining backgrounds are from $Q C D, W / Z$ production with heavy flavor, top and dibosons.

The $Z \boldsymbol{Z H} \rightarrow l^{+} \boldsymbol{l}^{-}-\mathbf{b}$ event selection starts by requiring two isolated high-P leptons with an invariant mass consistent with a $Z$ boson, $76<M_{l l}<106 \mathrm{GeV} / c^{2}$. Each event must contain two or three jets with $E_{T}>15 \mathrm{GeV}$ and $|\eta|<2$ and pass a missing transverse energy cut of $\phi_{T}<50 \mathrm{GeV}$, designed to reduce $t \bar{t}$ background. Finally, at least one jet must be identified as a $b$ quark by the secondary vertex algorithm. The dijet invariant mass is obtained using the $b$-tagged jet and the highest- $E_{T}$ non-tagged jet. The major backgrounds are from $Z$ plus heavy flavor and mistags.

The total acceptance per channel for identifying $W H / Z H$ events, reported in 
Table 1, was calculated from data and a SM simulation of Higgs production, ${ }^{5}$ with a total systematic uncertainty in the range of 20-30\%. The number of events observed in the data after the $V b \bar{b}$ selection is consistent with the predicted background.

A combined upper limit on the Higgs production cross section is calculated from a total likelihood function which is the product of the individual likelihoods for each channel. These are binned likelihoods of the dijet mass spectrum including background expectations and signal templates for different Higgs masses. All backgrounds are gaussian constraint within their respective uncertainties, except the $q q b \bar{b}$ QCD background which is allowed to float in the fit. The correlations between the systenatic uncertainties in the acceptance are taken into consideration with the $b$-tagging efficiency considered to be $100 \%$ correlated between all channels. The $95 \%$ C.L. upper limits on the $V H$ production cross section times the $B R(H \rightarrow b \bar{b})$ were calculated for the Standard Model Figgs masses of 90,110 and $130 \mathrm{GeV} / c^{2}$ and are summarized in Figure 1. The combined limit on $\sigma(p \bar{p} \rightarrow V H) \times \mathrm{BR}(\mathrm{H} \rightarrow \mathrm{b} \overline{\mathrm{b}})$ for the above Higgs masses is respectively $8.2 p b, 7.8 p b$ and $7.4 p b$.
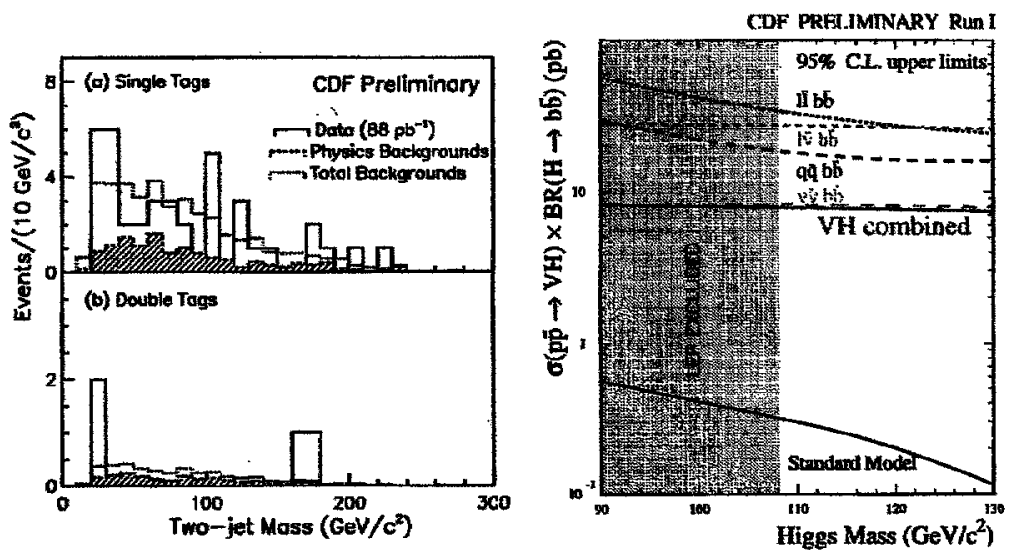

Fig. 1. Left plot: Invariant dijet mass distribution in the $Z H \rightarrow \nu \bar{\nu} b \bar{b}$ channel. Comparison of the data to the background expectations. (a) singled-tagged events and (b) double-tagger events. Right plot: Upper limit on the $V H$ production cross-section times the $H \rightarrow b \bar{b}$ branching ratio. The 95\% C.L. limits for each individual channel and the combined limit are compared to the SM expectation.

\section{References}

1. B. Pietrzyk for the LEPEWWG, in Proceedings of 30th ICHEP, Osaka, Japan, 2000.

2. F. Abe et al., Phys. Rev. Lett. 74 (1995) 2626; Phys. Rev. D 53 (1996) 1051.

3. F. Abe et al., Phys. Rev. Lett. 79 (1997) 3819.

4. F. Abe et al., Phys. Rev. Lett. 81 (1998) 5748.

5. T. Sjöstrand, Comput. Phys. Commun. 82 (1994) 74. 